\title{
PREPRINT
}

RESEARCH ARTICLE

\section{A dynamic occupancy model for interacting species with two spatial scales}

\section{Authors:}

Eivind Flittie Kleiven ${ }^{1}$, Frederic Barraquand ${ }^{2,3}$, Olivier Gimenez ${ }^{4}$, John-André Henden ${ }^{1}$, Rolf Anker Ims ${ }^{1}$, Eeva M. Soininen ${ }^{1} \&$ Nigel Gilles Yoccoz ${ }^{1}$

${ }^{1}$ UiT - The Arctic University of Norway, Department of Arctic and Marine Biology, Troms $\varnothing$, Norway

${ }^{2}$ CNRS, Institute of Mathematics of Bordeaux, Talence, France

${ }^{3}$ Integrative and Theoretical Ecology, LabEx COTE, University of Bordeaux, Pessac, France

${ }^{4}$ CEFE, Université Montpellier, CNRS, EPHE, IRD, Universite Paul Valéry Montpellier 3, Montpellier, France

Corresponding author email: eivind.f.kleiven@uit.no

Running headline: A dynamic occupancy model for interacting species with two spatial scales 


\section{Abstract}

Occupancy models have been developed independently to account for multiple spatial scales and species interactions in a dynamic setting. However, as interacting species (e.g., predators and prey) often operate at different spatial scales, including nested spatial structure might be especially relevant in models of interacting species. Here we bridge these two model frameworks by developing a multi-scale two-species occupancy model. The model is dynamic, i.e. it estimates initial occupancy, colonization and extinction probabilities - including probabilities conditional to the other species' presence. With a simulation study, we demonstrate that the model is able to estimate parameters without bias under low, medium and high average occupancy probabilities, as well as low, medium and high detection probabilities. We further show the model's ability to deal with sparse field data by applying it to a multi-scale camera trapping dataset on a mustelid-rodent predator-prey system. The field study illustrates that the model allows estimation of species interaction effects on colonization and extinction probabilities at two spatial scales. This creates opportunities to explicitly account for the spatial structure found in many spatially nested study designs, and to study interacting species that have contrasted movement ranges with camera traps.

Keywords: co-occurrence, multi-scale, occupancy, predator-prey, spatial, species interactions

\section{Introduction}

Much of the data available to ecologists focus on species occurrence, which in turn have sparked the development of statistical models to analyse such data (Bailey et al., 2014). Due to their ability to model species occurrence while accounting for imperfect detection and therefore unobserved species, occupancy models have become widely used in ecology (Bailey et al., 2014; GuilleraArroita, 2017). Initial formulations of occupancy models estimated species occupancy across mul- 
tiple sites that were assumed to be spatially independent (MacKenzie et al., 2002). However, this assumption is rarely met in the field (Johnson et al., 2013), and failing to account for spatial dependencies will lead to overconfidence in estimated uncertainties, and might even reverse the sign of the estimated slope of relationships (Guélat \& Kéry, 2018).

There are numerous extensions of occupancy models to incorporate spatial dependencies. In static occupancy models, occupancy can be made dependent on the occupancy probability of neighboring sites (Bled et al., 2011; Eaton et al., 2014; Yackulic et al., 2014; Broms et al., 2016), while in dynamic models (i.e., models that explicitly estimate change over time), colonization probability can be made a function of occupancy probability of nearby sites. Spatial dependencies may be formulated as explicit functions of distance or connectivity between sites (Sutherland et al., 2014; Chandler et al., 2015), or in the form of random spatial effects (Johnson et al., 2013; Rota et al., 2016b).

Data from many ecological studies exhibit multiple nested spatial scales, which mirror the fact that population dynamics result from different processes occurring at multiple scales (Baumgardt et al., 2019). Accordingly, recently developed multi-scale occupancy models enable analyses of data from designs with such a hierarchy of spatial scales (Nichols et al., 2008; Aing et al., 2011; Mordecai et al., 2011; Kéry \& Royle, 2015; Smith \& Goldberg, 2020), and can be extended to dynamic versions to estimate colonization and extinction probabilities (Tingley et al., 2018).

A parallel development of occupancy models -dynamic multi-species models- addresses how interacting species co-occur over time (MacKenzie et al., 2004; Waddle et al., 2010; Richmond et al., 2010; Rota et al., 2016a; MacKenzie et al., 2017; Fidino et al., 2019; Marescot et al., 2020). These models have great potential to increase our knowledge of species interactions. However, interacting species in general, and predators and prey in particular, often move at different spatial scales (de Roos et al., 1998; Fauchald et al., 2000). Incorporating the multiple spatial scales of interacting species would therefore likely improve estimation and reduce bias in the estimated slope of relationships between parameters and covariates or state variables. Moreover, it allows 
for investigation of species interactions on multiple spatial scales, that may represent different ecological processes.

Here we build on dynamic multi-species models by MacKenzie et al. (2017) and Fidino et al. (2019), as well as the dynamic multi-scale occupancy model by Tingley et al. (2018) to develop a multi-scale dynamic two-species occupancy model. In this model, initial occupancy, colonization and extinction probabilities are estimated at two spatial scales, i.e. both at site level and at a regional level, spanning a cluster of sites. After describing the model, we perform a simulation study to investigate potential issues of bias and precision under different scenarios. Finally, we exemplify the usefulness of this approach for real data, by applying the model to a multi-scale camera trapping dataset of a mammalian predator-prey system.

\section{Methods}

\subsection{Data structure and latent ecological states}

The spatial setup and model structure for this multi-scale occupancy model is illustrated in Figure 1. Compared to the classical occupancy model, we have added an additional spatial level so that we have $b \in\{1, \ldots, B\}$ blocks, each of which contains $k \in\{1, \ldots, K\}$ sampling sites (see Fig. 1). Each sampling site is surveyed over $t \in\{1, \ldots, T\}$ sampling occasions (hereafter primary occasions). Each primary occasion is sampled $J$ times, $j \in\{1, \ldots, J\}$ being the index of the survey (hereafter secondary occasion). For each secondary occasion, a species is either recorded as observed or unobserved. For ease of presentation we assumed that sampling is equal among all sites and blocks, but this is not a requirement of the model. 


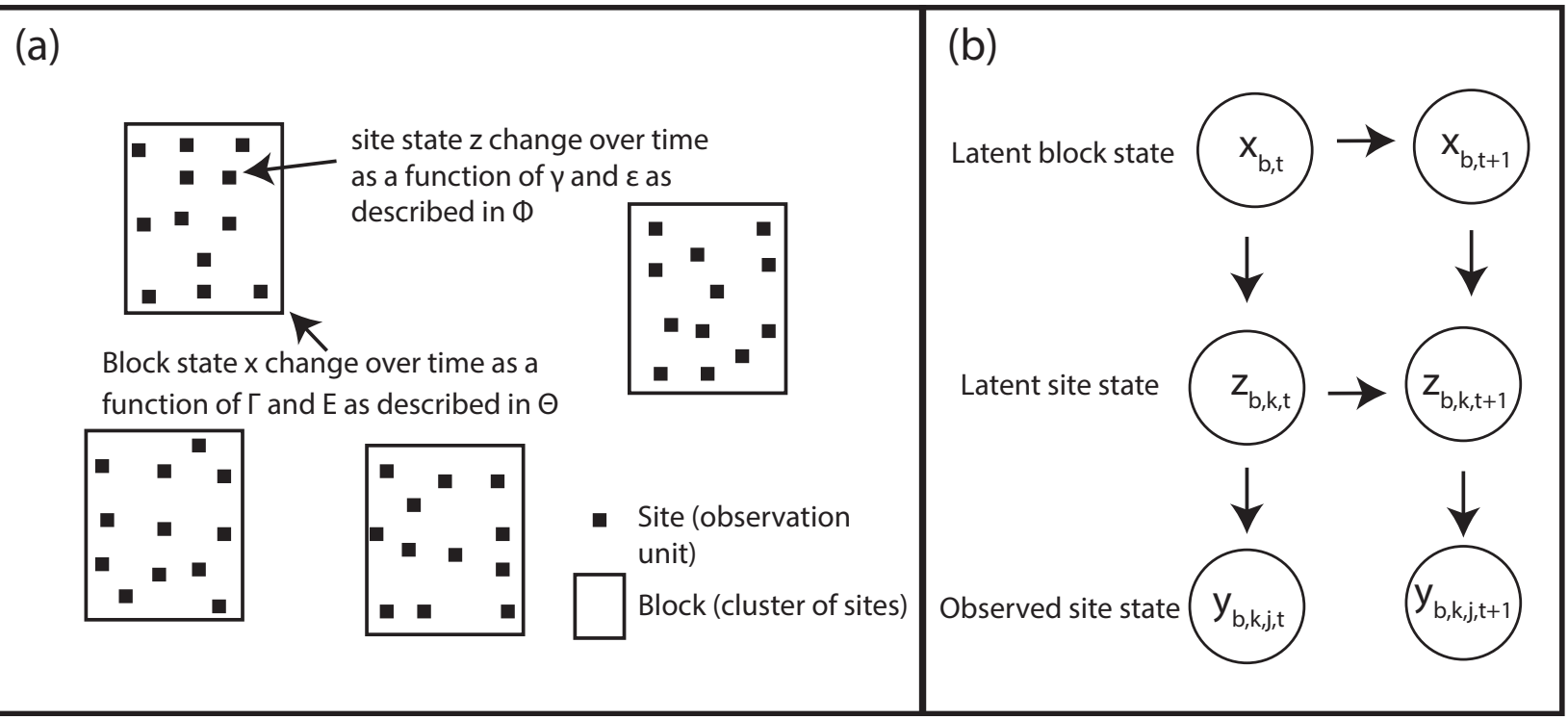

Figure 1: Conceptual diagram of the design and model structure. Panel (a) describes the multi-scale structure of the design, data sampling and state variables (probabilities) with clusters of sites nested in 4 blocks. Parameters $\gamma$ and $\varepsilon$ denote site level colonization and extinction probabilities, respectively, and where $\Phi$ denote the site transition probability matrix. Parameters $\Gamma$ and $E$ are the block level colonization and extinction probabilities, respectively, and where $\Theta$ denotes the block level transition probability matrix. The diagram in panel (b) shows the conditional dependencies among the state variables in the model. The index $b$ indicates a given block, $k$ a given site, $t$ a given primary occasion and $j$ a given secondary occasion.

Following MacKenzie et al. (2017) we describe the state of each site (k) during each primary occasion ( $\mathrm{t}$ ) in a given block (b) with a latent variable $z_{b, k, t}$. Considering a two species model, any given site can then either be unoccupied (U), occupied by species A only (A), occupied by species B only (B) or occupied by both species A and B (AB). In this model we also consider an ecological process on the block level by describing a latent block state, $x_{b, t}$, that can take on any of the same four states as the latent site level state (U, A, B, or AB).

\subsection{Transition model}

After initial states have been modelled as a random categorical variable (see Appendix S1 for details), transitions between states are modelled with the transition probability matrices $\Theta$ for the 
block level and $\Phi$ for the site level, with the latter depending on the block state. For simplicity it is assumed that the transition parameters (colonization and extinction) are equal for all sites, blocks and time steps. It is further assumed that the site colonization $(\gamma)$ and extinction $(\varepsilon)$ probabilities are dependent on the site state in the previous time step $\left(z_{k, b, t-1}\right)$ and the block state in the same time step $\left(x_{b, t}\right)$. The former is assumed because site level colonization is only possible whenever the given species is already present in the block. Because site transition probabilities are dependent on the block state in the same time step $\left(\Phi\left(x_{b, t}\right)\right)$, it is possible that both a block and sites within that block are colonized in the same time step. However, because sites do not cover all available habitat within the block, blocks are not forced to go extinct even though all sites within that block are extinct. Hence, the model allows for a block to remain occupied even when all of its sites go extinct. On the other hand, when a block goes extinct, all of its sites are forced to go extinct (i.e. if $x_{b, t}=\mathrm{U}$ then $\left.z_{b, k, t}=\mathrm{U}\right)$.

The transition probability matrix $(\Theta)$ for blocks $(b)$ can be written as

To state

$$
\begin{aligned}
& \begin{array}{llll}
U & A & B & A B
\end{array}
\end{aligned}
$$

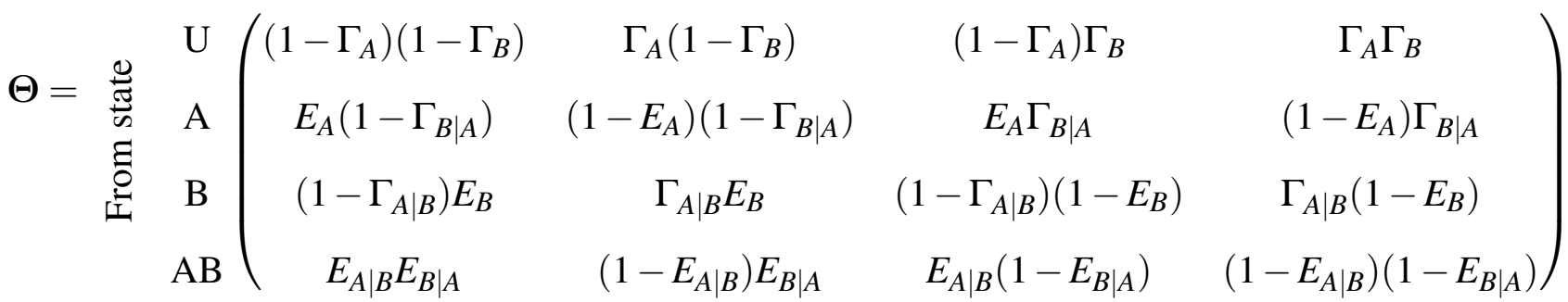

Since the site transition probabilities depend on the block level state $\left(x_{b, t}\right)$ we create one site transition matrix for each possible block state in the following way: 
To state

$$
\begin{aligned}
& \begin{array}{llll}
U & A & B & A B
\end{array}
\end{aligned}
$$

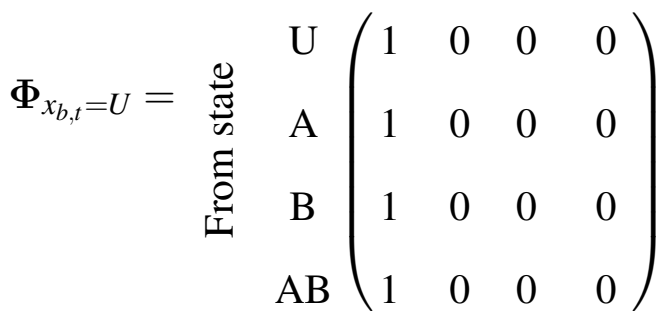

To state

$$
\begin{aligned}
& \begin{array}{llll}
U & A & B & A B
\end{array}
\end{aligned}
$$

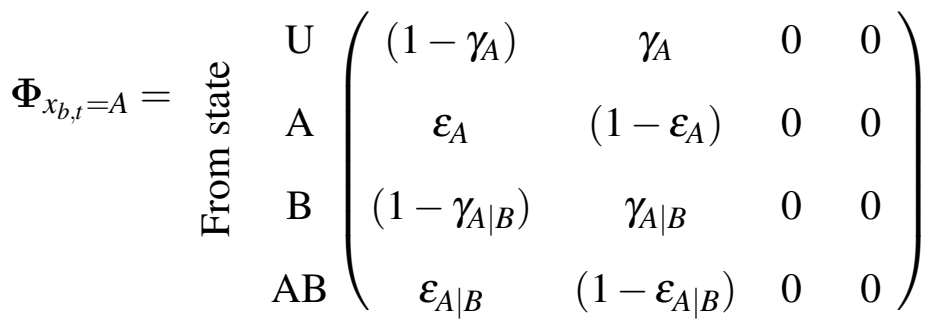

To state

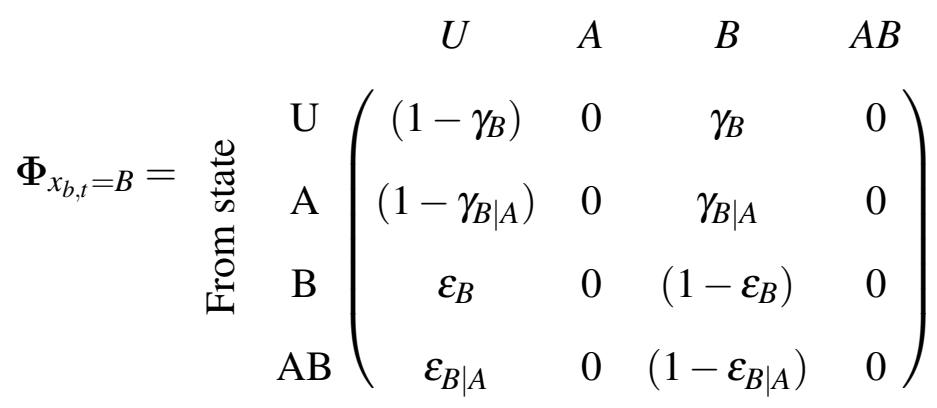


To state

$$
\begin{aligned}
& \begin{array}{llll}
U & A & B & A B
\end{array}
\end{aligned}
$$

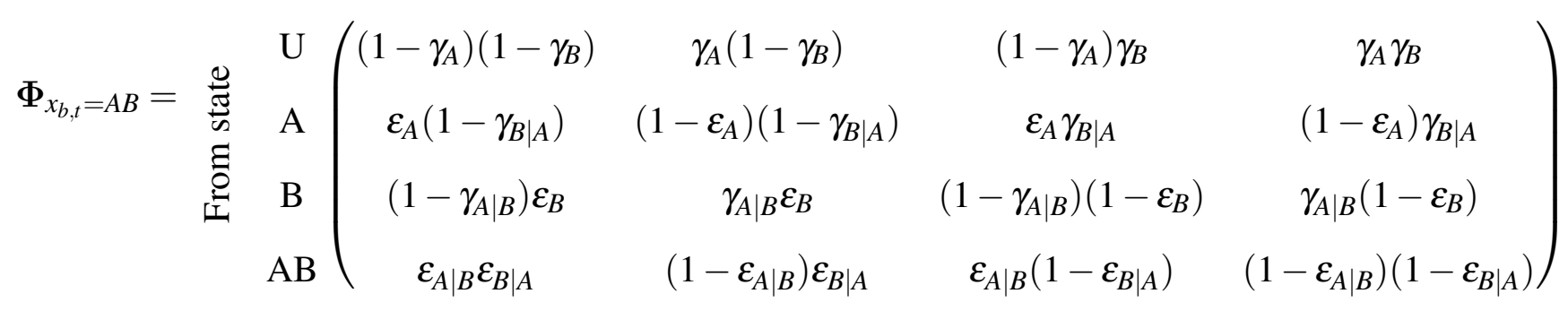

Then the full model for both block- and site level states can be written as

$$
\begin{array}{r}
x_{b, t} \mid\left(x_{b, t-1}, \Gamma, E\right) \sim \operatorname{Categorical}\left(\Theta_{x_{b, t-1}, \bullet}\right) \text { for } t=2, \ldots, T \\
z_{k, b, t} \mid\left(x_{b, t}, z_{k, b, t-1}, \gamma, \varepsilon\right) \sim \operatorname{Categorical}\left(\Phi\left(x_{b, t}\right)_{z_{k, b, t-1}, \bullet}\right) \text { for } t=2, \ldots, T .
\end{array}
$$

The indices $x_{b, t-1}$ describe the specific row in $\Theta$ which has value $x_{b, t-1}$ and $z_{k, b, t-1}$ the row in $\boldsymbol{\Phi}$ which has value $z_{k, b, t-1}$, while $\bullet$ refers to all columns of that row.

\subsection{Detection model}

All observations on which the model relies are done at the site level. Hence, the detection probability can be modelled similarly to other two-species occupancy models. Models to estimate detection probabilities of one species dependent on the presence (Rota et al., 2016a) or the detection of the other species (Miller et al., 2012; Fidino et al., 2019) exist. However, for simplicity it is here assumed that detection of each species is independent of both the presence and detection of the other species. For simplicity, it is also assumed in the simulation study that the detection probability is constant over sites, blocks and temporal occasions. However, note that a temporal covariate is 
added to the detection model in the empirical case study (see related section below). Let $p_{A}$ and $p_{B}$ be the detection probabilities of species $\mathrm{A}$ and $\mathrm{B}$ at a given site, the detection probability matrix ( $\lambda$ ) can then be defined as

$$
\begin{aligned}
& \text { Observed state }
\end{aligned}
$$

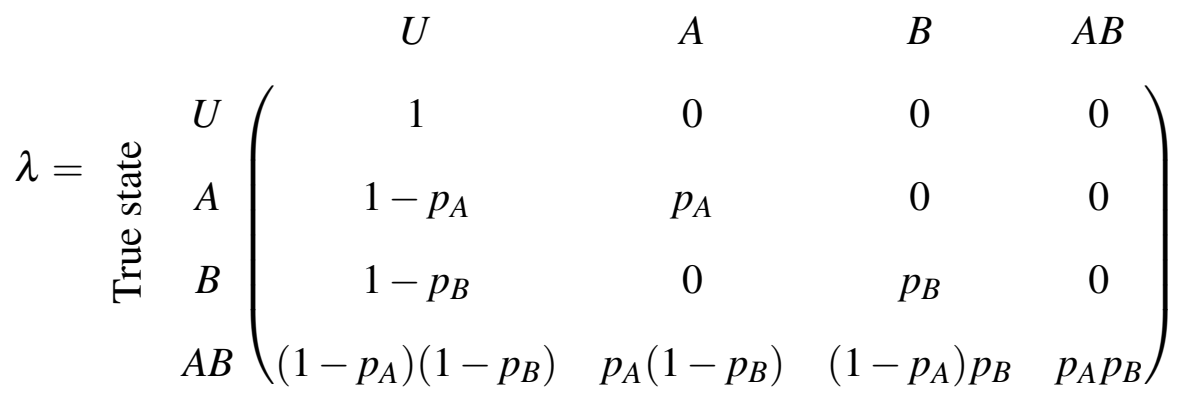

The observation at site $k$ in block $b$ at visit $j$ during time $t\left(y_{k, b, j, t}\right)$ can then be described by the following equation:

$$
y_{k, b, j, t} \mid\left(x_{b, t}, z_{k, b, t}\right) \sim \text { Categorical }\left(\lambda_{z_{k, b, t}, \bullet}\right)
$$

with $z_{k, b, t}$ being the chosen row of the detection matrix $(\lambda)$ to draw the observed state. We note that for limited data the detection probability matrix could be simplified further by estimating an independent detection probability for each observable state (Marescot et al., 2020).

\subsection{Simulation study}

We conducted a simulation study to evaluate the performance of our model by examining potential issues of bias and variance in parameter estimates of colonization, extinction and detection. The spatiotemporal structure of the simulated data was largely inspired by our empirical case study (see 
section below for a more detailed explanation). Hence, we simulated data for 8 blocks, each containing 12 sites. We chose weeks as primary occasions and days within each week as secondary occasions corresponding to the expected rate of the dynamics of the empirical case study (Andreassen \& Ims, 2001). We simulated data for 50 weeks (approximately one year). The chosen parameters values were also inspired by the predator-prey case study (e.g. $E_{A}<E_{B}$. See all parameter values in Appendix S1). To investigate how contrasting scenarios affected bias of parameters, we simulated data where both species had low (lo), medium (mo) and high (ho) average occupancy probability. In addition we simulated data where both species had low (ld), medium (md) and high (hd) detection probabilities (for details, see Appendix S1). For each scenario, we simulated and analyzed 50 replicate datasets. The models were analyzed in a Bayesian framework using the JAGS software (Plummer, 2003) with the jagsUI package (Kellner, 2015) in R V 4.0 .3 (R Core Team, 2020). We specified uniform prior distributions $(\sim$ Uniform $(0,1))$ for the colonization and extinction probabilities. After a adaptation phase of 1000 and a burn-in of 0 , the model was run for 5000 iterations with a thinning of 10 . Convergence was assessed by having a $\hat{R}<1.1$ for all key parameters (Gelman et al., 2013) and from graphical investigation of traceplots for all parameters.

\subsection{Empirical case study}

To illustrate the real-world applicability of our model, we used a camera trap dataset from the longterm monitoring program COAT (Climate-ecological Observatory of the Arctic Tundra)(Ims et al., 2013). The camera trapping program targets small rodents and their small mustelid predators, both of which form a functional group. Here rodents (grey-sided vole Myodes rufucanus, tundra vole Microtus oeconomus and Norwegian lemming Lemmus lemmus) constitute prey (group A), while mustelids (stoat Mustela erminea and least weasel Mustela nivalis) constitute predators (group B). The camera traps, described by Soininen et al. (2015), are active year-round in arctic tundra habitats $\left(70^{\circ} 20^{\prime} \mathrm{N} 29^{\circ} 38^{\prime} \mathrm{E}\right)$. The sampling design has a multi-scale structure, where sites (camera 
traps), are spaced $>300 \mathrm{~m}$ apart, but clustered in blocks of 11 to 12 cameras covering two different habitats, snowbeds and hummock tundra (see Appendix S1). This corresponds to movement ranges of rodents and mustelids, where sites represent independent samples of rodent presence and blocks represent independent samples of mustelid presence (Hellstedt \& Henttonen, 2006). Mustelids almost only occurred in the snowbed habitat, hence we chose to focus only on these sites, reducing the number of cameras to 5 - 6 per block. The dataset consists of a total of 8 blocks. As the rodents are known to exhibit fast local scale colonization-extinction dynamics (Andreassen \& Ims, 2001) we here define a primary occasion as one week (i.e. 7 days) and secondary occasions as the days within that week.

All images of the two functional groups (i.e. rodents and mustelids) were identified by means of an algorithm for automatic classification of camera trap images. The MLWIC R-package (Tabak et al., 2019) was used to train the algorithm (see Appendix S1 for more details). The resulting dataset describes, for each secondary occasion (day), if each functional group was detected or not. The four states of sites and blocks thus correspond to $\mathrm{U}=$ none of the species are observed, $\mathrm{A}$ $=$ only rodents observed, $\mathrm{B}=$ only mustelids observed, or $\mathrm{AB}=$ both rodents and mustelids are observed. To analyze this data, we included all weeks from the monitoring commenced in 2015, making a total of 203 weeks. The blocks where established at different times, with only 4 blocks established at the beginning of the study and 4 more blocks included in 2018 (see Appendix S1). This resulted in inclusion of missing data since more then half of the sites were only observed for the last 47 weeks.

This study system has a strong seasonality that we needed to account for. Accordingly, we used a binary covariate defining summer as between 1st of July and 1st of November while the remaining months were defined as winter. This variable was then included as a covariate on detection probabilities $\left(p_{A}\right.$ and $\left.p_{B}\right)$ through a logit-link function. The model was analyzed similarly to the simulation model. Prior distributions of the colonization and extinction probabilities was specified as for the simulation study $(\sim \operatorname{Uniform}(0,1))$. For the detection probability the prior 
distribution for the intercept was specified as $\operatorname{logit}(\operatorname{Uniform}(0,1))$ and for the slope parameters as Uniform $(-10,10)$. However, to reach convergence with the real dataset we needed 25000 steps of adaptation, a thinning of 20 and to run the model for 100000 iterations.

\section{Results}

\subsection{Simulation study}

All colonisation and extinction probabilities at both spatial scales were estimated without bias for the two most data-rich scenarios (high detection probability and high occupancy probability) (Fig. 2). For the rest of the scenarios, most parameters were also estimated almost without bias. However, for $E_{A B}$ there was a positive bias under 3 scenarios, for $\Gamma_{A B}$ there was a small negative bias under 3 scenarios and for $\gamma_{A}$ and $\gamma_{B}$ there was a small positive bias for 4 scenarios (see Fig. 2). Both detection probabilities $\left(p_{A}\right.$ and $\left.p_{B}\right)$ were estimated without any apparent bias. The initial values were also estimated without any obvious bias except for $\psi_{A B}$ under the low occupancy scenario, where it seems to be a small positive bias (see Appendix S1). 

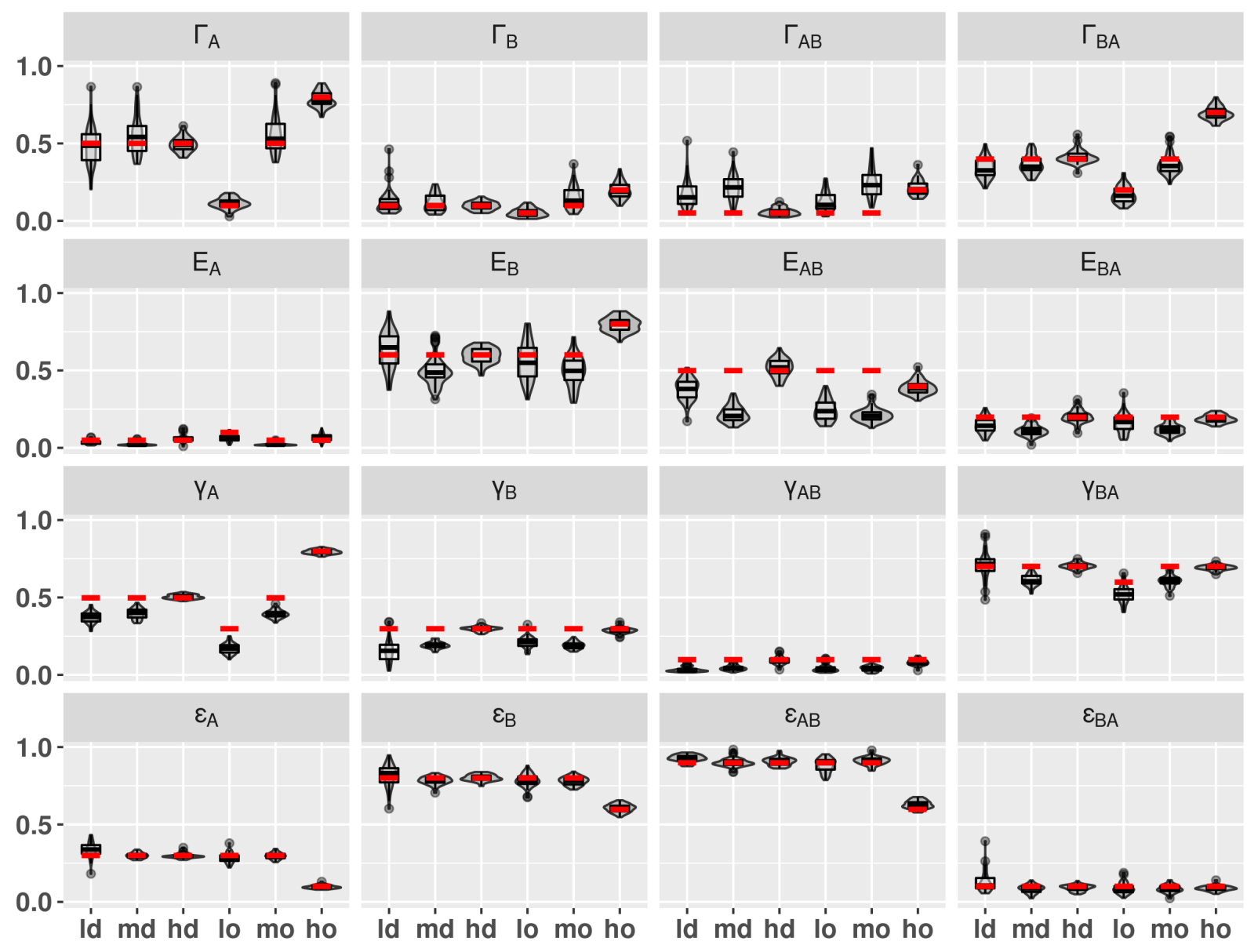

Id ma h'd lo mo h'o

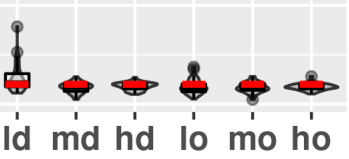

Figure 2: Violin plot and boxplot of the posterior means of site and block colonization $(\gamma$ and $\Gamma)$ and extinction probabilities ( $\varepsilon$ and $E$ ), from 50 simulation replicates. The red bar indicates the true parameter values. The $\mathrm{x}$-axis displays the 6 different data scenarios: low, medium and high detection probability of both species (ld,md,hd) and low, medium and high average site occupancy probability of both species (lo,mo,ho).

\subsection{Empirical case study}

Rodents generally had a higher detection probability than mustelids (for detection probabilities, see Appendix S1). Both functional groups (i.e. rodents and mustelids) were less detectable during winter, but the seasonal effect were much stronger for the mustelids with detection probability close to zero during winter. 
For rodents, we found a clear indication of mustelid impacts at the site level, as mustelid presence decreased site level colonization probability from 0.116 to 0.003 (a decrease of 0.11 with $90 \%$ CI (0.08-0.14)), and increased extinction probability from 0.076 to 0.595 (an increase of 0.52 with 90\% CI (0.29-0.77)) (for colonization/extinction probabilities, see Fig. 3). Block extinction of rodents appeared unaffected by the presence of mustelids in the block. For mustelids, we found no positive impacts of rodents at the site level. It rather seemed to be the opposite, as mustelid colonization decreased from 0.230 to 0.062 (a decrease of 0.17 with $90 \% \mathrm{CI}(0.08-0.27)$ ) when rodents were present. At the block level, however, mustelid colonization probability increased from 0.011 to 0.076 (an increase of 0.07 with $90 \% \mathrm{CI}(0.03-0.11)$ ) when rodents were already present in the block. This suggests that different factors are controlling the colonization of mustelids at the two different spatial scales. Overall, these results indicate a strong predator-prey interaction, observed for each functional group at the spatial scale which was targeted by the study design. We note, however, that three of the parameters (i.e. block level colonization of rodents when mustelids were present $\left(\Gamma_{A B}\right)$, block level extinction of mustelids alone $\left(E_{B}\right)$, and site level extinction of mustelids when rodents were present $\left.\left(\varepsilon_{B A}\right)\right)$ were estimated with a large uncertainty. This makes ecological inference about these parameters impossible (See Tables S4 and S5 in Appendix S1 for precise estimates of all colonization and extinction probabilities, and the differences between them, with corresponding credible intervals). 


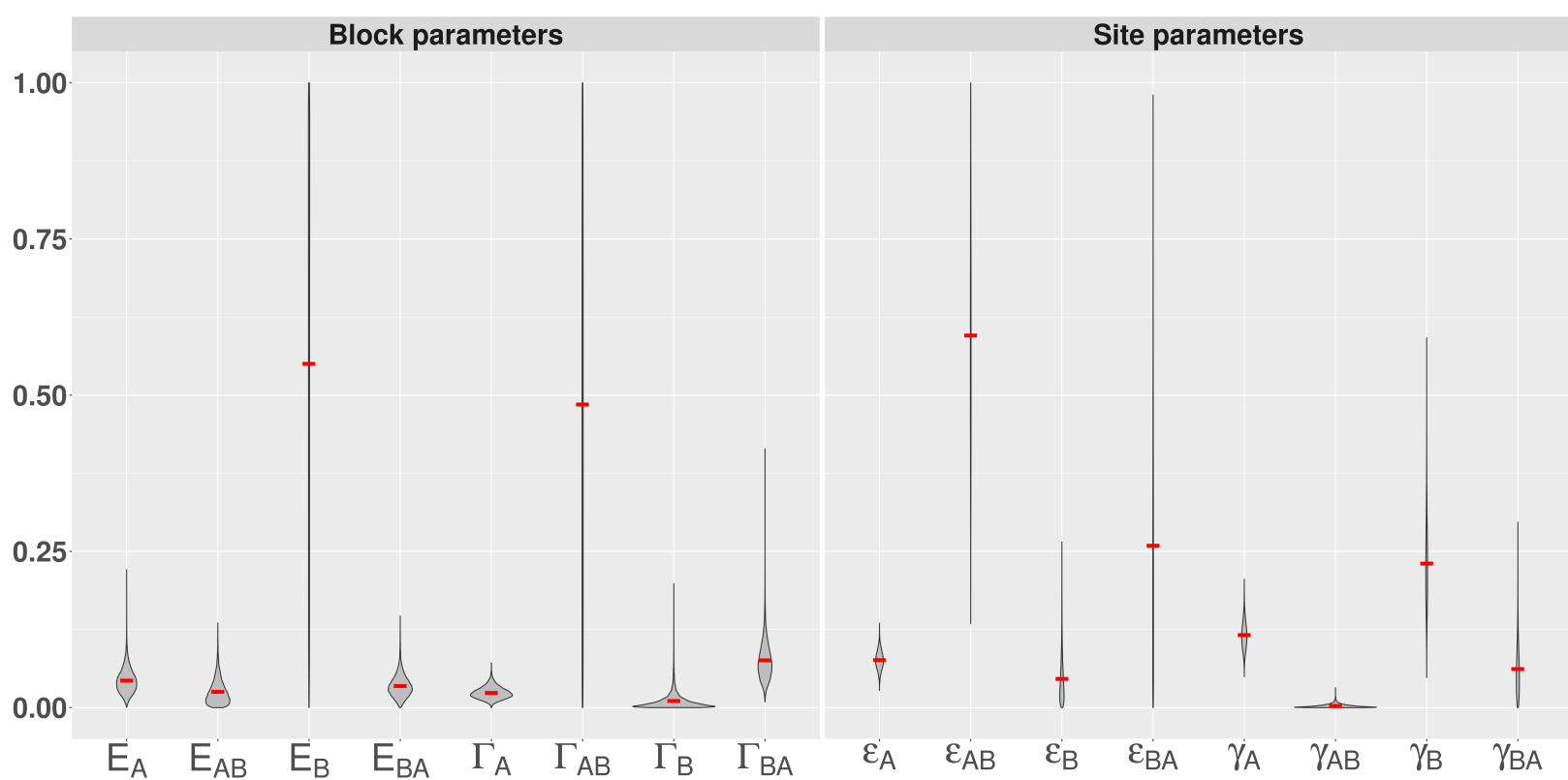

Figure 3: Violin plots of the estimated posterior distribution of site and block colonization ( $\gamma$ and $\Gamma$ ) and extinction probabilities ( $\varepsilon$ and $E$ ) for the case study. Subscript $A$ denotes small rodents and $B$ denote small mustelids. Red bars indicate posterior means.

\section{Discussion}

We have constructed a dynamic multi-scale occupancy model for interacting species. Through simulations, we demonstrate that the model is able to produce mostly unbiased estimates of colonization and extinction parameters under different scenarios of average presence and detection. We find the current extension of the dynamic multi-species occupancy framework to nested spatial scales useful for two reasons. First, it is possible to explicitly account for the spatial structures found in many spatially nested study designs. This should lead to better estimates of uncertainty and reduce bias in parameter estimates. Second, it makes it possible to investigate the joint colonization and extinction dynamics of species pairs that have contrasting movement ranges, where the daily movement range of one species (e.g., the predator) is equivalent to the dispersal distance for another species (e.g., the prey). Analysing data from a multi-scale monitoring program of 
two species with contrasted movements ranges (cf. our mustelid-rodent case study) with a single scale model would make little sense. Not only would this violates the assumption of spatial independence, but it would also compromise inference in terms of ecologically meaningful model parameters. This is because the two spatial scales represent different ecological processes: the spatial scale of short distance colonization for rodents (between sites) corresponds to the scale of within-home range movements for mustelids (e.g. foraging), while the spatial scale of long distance colonization for rodents (between blocks) correspond for mustelids to the scale of between-home range movements (e.g. changing territories). Our work therefore helps to bridge the gap between the separate developments of multi-scale dynamic occupancy models (Tingley et al., 2018) and dynamic multi-species occupancy models (MacKenzie et al., 2017).

The simulation study shows that all model parameters are estimated without bias in the two most data-rich scenarios (high occupancy and high detection). In data-poor scenarios, some small biases appeared, especially for the block extinction probability of $\mathrm{A}$ when $\mathrm{B}$ is present $\left(E_{A B}\right)$. A likely explanation is that when few blocks are occupied with both species, there are also few blocks that have the potential to go extinct. The contrast in bias between the 6 data scenarios suggests that this spatio-temporal study design may be at the lower end of the quantity of data needed to produce unbiased results. This is a consequence of working with species with low detection and/or occupancy probability. However, we have considered vague priors, and more informative priors may lead to improved estimation. Furthermore, the block-level parameters appear to vary more between models than the site-level parameters. This could result from all observations coming from the site-level, with the block-level parameters thus depending on the reconstruction of two latent states. Also, the nested study design has logically more sites than blocks, providing more data to estimate the site-level parameters than the block-level parameters. We therefore encourage further work on the data requirements of similarly complex multi-scale occupancy models.

Our real-world case study incorporates some of the classical empirical challenges that ecologists have to deal with. The dataset has a high proportion of missing data and detection probability 
differs between the functional groups. In addition, the dataset comes from an ecosystem with both strong seasonality and multi-year cyclicity, which likely affects the detection, colonization and extinction probabilities. Moreover, we use functional groups instead of species, potentially adding some unexplained variability in the data. Also, we treated blocks as if they were identical, which is likely to be an oversimplification. Even without addressing these challenges specifically in the model (with the exception of a seasonal covariate on detection probability), the model seems to be able to identify most parameters. However, it is evident that some parameters $\left(\varepsilon_{A B}, \Gamma_{A B}\right.$ and $\left.E_{B}\right)$ are estimated with large uncertainties, to a degree where it limits the ecological inference that can be made (Fig. 3). Our empirical case study appears thus to constitute the minimal data requirements for this model. It is likely that a longer time series would increase parameter precision (GuilleraArroita et al., 2014), especially in the case study system where the population dynamics are ruled by 4-year population cycles.

Despite these challenges, the model gave clear evidence of a strong predator-prey interaction between mustelids and rodents (Hanski et al., 1993; Norrdahl \& Korpimäki, 2000). Presence of rodents in a block increased the weekly block colonization probability of mustelids, while on site level we found no positive effect of rodents on the colonization probability of mustelids. This suggests that medium-range movements of mustelids are affected by rodents, while other factors seem to control small-scale mustelid movements related to foraging. On the site level, mustelid presence both reduced drastically rodent colonization probability and increased rodent extinction probability, which is probably due to direct effects (killing) and indirect effects (predator avoidance) of predation by mustelids. These results are perfectly in line with the predator-prey hypothesis for rodent population cycles. Most of the current observational evidence for this hypothesis comes from indirect observations of mustelids (snow tracks and winter nests Gilg et al., 2003; Korpela et al., 2014 and spatially aggregated rodent counts: we provide here some support for the predation hypothesis from direct observations at spatial and temporal scales commensurate to those of theoretical models. 
We note that numerous extensions to this model could be possible. Although we only included a simple discrete covariate (season) for the detection probabilities in the case study, the model could be extended to include both temporal and spatial covariates on initial occupancy, colonization and extinction probabilities following earlier multi-species occupancy models (Rota et al., 2016a; Fidino et al., 2019; Kery \& Royle, 2020). Our model also has the potential to be extended to more species (similarly to models by Rota et al. 2016a and Fidino et al. 2019) or spatial levels. However, adding many more species, or in other ways complicate the model further, would probably require regularization (shrinkage of some parameters) or variable selection (Hutchinson et al., 2015; McElreath, 2015).

To conclude, we developed a dynamic occupancy model for interacting species at two spatial scales, which estimates initial occupancy, colonization and extinction probabilities as well as detection probabilities. Applied to northern rodent population dynamics, the model provided some evidence in support of the predation hypothesis as an important contributor to rodent population cycles, and was able to further pinpoint that interactions between predators and prey in fact arise from processes occurring at two nested spatial scales.

\section{Acknowledgments}

We thank H. Böhner for the invaluable help with classifying the camera trap images, M.Murphy, L.E. Støvern, I. Jensvoll and S. Killengreen for help with installing the camera trap monitoring design, M.L. Dahle and J.E. Knutsen for help with checking cameras and J.P. Mellard for improving the language in this manuscript. This work is a part of the projects COAT (www.coat.no), supported by the RCN (project nr. 245638), and COAT-Tools+, supported by TFS and UiT. OG was funded by the French National Research Agency (grant ANR-16-CE02-0007). 


\section{Author contributions}

Eivind F. Kleiven: Conceptualization (equal); Data curation (supporting); Formal analysis (lead); Investigation (equal); Methodology (equal); Project administration (lead); Software (lead); Validation (equal); Visualization (lead); Writing - original draft (lead): Writing - review \& editing (lead). Frederic Barraquand: Conceptualization (equal); Formal analysis (supporting); Investigation (equal); Methodology (equal); Project administration (supporting); Software (supporting); Validation (equal); Visualization (supporting); Writing - original draft (supporting); Writing - review \& editing (equal). Olivier Gimenez: Conceptualization (equal); Formal analysis (supporting); Funding acquisition (lead); Methodology (supporting); Validation (supporting); Visualization (supporting); Writing - review \& editing (equal). John-André Henden: Conceptualization (equal), Formal analysis (supporting); Methodology (supporting); Writing - review \& editing (equal). Rolf A. Ims: Conceptualization (equal), Funding acquisition (lead); Investigation (equal); Writing review \& editing (equal). Eeva M. Soininen: Conceptualization (equal); Data Curation (lead); Funding acquisition (lead); Investigation (equal); Project administration (supporting); Visualization (supporting); Writing - original draft (supporting); Writing - review \& editing (equal). Nigel G. Yoccoz: Conceptualization (equal), Funding acquisition (lead); Formal analysis (supporting); Methodology (supporting); Writing - review \& editing (equal).

\section{Data Availability}

All data and code used in this manuscript are available at UiT Open Research Data (doi.org/10.18710/ZLW59W). 


\section{References}

Aing, C., Halls, S., Oken, K., Dobrow, R. \& Fieberg, J. (2011). A bayesian hierarchical occupancy model for track surveys conducted in a series of linear, spatially correlated, sites. Journal of Applied Ecology, 48, 1508-1517.

Andreassen, H.P. \& Ims, R.A. (2001). Dispersal in patchy vole populations: Role of patch configuration, density dependence, and demography. Ecology, 82, 2911-2926.

Bailey, L.L., MacKenzie, D.I. \& Nichols, J.D. (2014). Advances and applications of occupancy models. Methods in Ecology and Evolution, 5, 1269-1279.

Baumgardt, J.A., Morrison, M.L., Brennan, L.A. \& Campbell, T.A. (2019). Developing rigorous monitoring programs: Power and sample size evaluations of a robust method for monitoring bird assemblages. Journal of Fish and Wildlife Management, 10, 480-491.

Bled, F., Royle, J.A. \& Cam, E. (2011). Hierarchical modeling of an invasive spread: the eurasian collared-dove streptopelia decaocto in the united states. Ecological Applications, 21, 290-302.

Broms, K.M., Hooten, M.B., Johnson, D.S., Altwegg, R. \& Conquest, L.L. (2016). Dynamic occupancy models for explicit colonization processes. Ecology, 97, 194-204.

Chandler, R.B., Muths, E., Sigafus, B.H., Schwalbe, C.R., Jarchow, C.J. \& Hossack, B.R. (2015). Spatial occupancy models for predicting metapopulation dynamics and viability following reintroduction. Journal of Applied Ecology, 52, 1325-1333.

de Roos, A.M., McCauley, E. \& Wilson, W.G. (1998). Pattern formation and the spatial scale of interaction between predators and their prey. Theoretical Population Biology, 53, 108 - 130.

Eaton, M.J., Hughes, P.T., Hines, J.E. \& Nichols, J.D. (2014). Testing metapopulation concepts: effects of patch characteristics and neighborhood occupancy on the dynamics of an endangered lagomorph. Oikos, 123, 662-676. 
Fauchald, P., Erikstad, K.E. \& Skarsfjord, H. (2000). Scale-dependent predator-prey interactions: The hierarchical spatial distribution of seabirds and prey. Ecology, 81, 773-783.

Fidino, M., Simonis, J.L. \& Magle, S.B. (2019). A multistate dynamic occupancy model to estimate local colonization-extinction rates and patterns of co-occurrence between two or more interacting species. Methods in Ecology and Evolution, 10, 233-244.

Gelman, A., Carlin, J.B., Stern, H.S., Dunson, D.B., Vehtari, A. \& Rubin, D.B. (2013). Bayesian data analysis. CRC press.

Gilg, O., Hanski, I. \& Sittler, B. (2003). Cyclic dynamics in a simple vertebrate predator-prey community. Science, 302, 866-868.

Guillera-Arroita, G. (2017). Modelling of species distributions, range dynamics and communities under imperfect detection: advances, challenges and opportunities. Ecography, 40, 281-295.

Guillera-Arroita, G., Lahoz-Monfort, J.J., MacKenzie, D.I., Wintle, B.A. \& McCarthy, M.A. (2014). Ignoring imperfect detection in biological surveys is dangerous: A response to 'fitting and interpreting occupancy models'. PLOS ONE, 9, 1-14.

Guélat, J. \& Kéry, M. (2018). Effects of spatial autocorrelation and imperfect detection on species distribution models. Methods in Ecology and Evolution, 9, 1614-1625.

Hanski, I., Turchin, P., Korpimäki, E. \& Henttonen, H. (1993). Population oscillations of boreal rodents: regulation by mustelid predators leads to chaos. Nature, 364, 232-235.

Hellstedt, P. \& Henttonen, H. (2006). Home range, habitat choice and activity of stoats (mustela erminea) in a subarctic area. Journal of Zoology, 269, 205-212.

Hutchinson, R.A., Valente, J.J., Emerson, S.C., Betts, M.G. \& Dietterich, T.G. (2015). Penalized likelihood methods improve parameter estimates in occupancy models. Methods in Ecology and Evolution, 6, 949-959. 
Ims, R., Jepsen J., U., Stien, A. \& Yoccoz, N.G. (2013). Science plan for COAT: Climate-ecological Observatory for Arctic Tundra. Fram Centre Report Series 1. Fram Centre, Norway.

Johnson, D.S., Conn, P.B., Hooten, M.B., Ray, J.C. \& Pond, B.A. (2013). Spatial occupancy models for large data sets. Ecology, 94, 801-808.

Kellner, K. (2015). jagsui: a wrapper around rjags to streamline jags analyses. R package version, 1.

Kéry, M. \& Royle, J.A. (2015). Applied Hierarchical Modeling in Ecology: Analysis of distribution, abundance and species richness in R and BUGS: Volume 1: Prelude and Static Models. Academic Press.

Kery, M. \& Royle, J.A. (2020). Applied Hierarchical Modeling in Ecology: Analysis of distribution, abundance and species richness in $R$ and BUGS: Volume 2: Dynamic and Advanced Models. Academic Press.

Korpela, K., Helle, P., Henttonen, H., Korpimäki, E., Koskela, E., Ovaskainen, O., Pietiäinen, H., Sundell, J., Valkama, J. \& Huitu, O. (2014). Predator-vole interactions in northern europe: the role of small mustelids revised. Proceedings of the Royal Society B: Biological Sciences, 281, 20142119.

MacKenzie, D.I., Bailey, L.L. \& Nichols, J.D. (2004). Investigating species co-occurrence patterns when species are detected imperfectly. Journal of Animal Ecology, 73, 546-555.

MacKenzie, D.I., Nichols, J.D., Lachman, G.B., Droege, S., Andrew Royle, J. \& Langtimm, C.A. (2002). Estimating site occupancy rates when detection probabilities are less than one. Ecology, 83, 2248-2255.

MacKenzie, D.I., Nichols, J.D., Royle, J.A., Pollock, K.H., Bailey, L. \& Hines, J.E. (2017). Occupancy estimation and modeling: inferring patterns and dynamics of species occurrence. Elsevier. 
Marescot, L., Lyet, A., Singh, R., Carter, N. \& Gimenez, O. (2020). Inferring wildlife poaching in southeast asia with multispecies dynamic occupancy models. Ecography, 43, 239-250.

McElreath, R. (2015). Statistical rethinking: A Bayesian course with examples in R and Stan. CRC press.

Miller, D.A.W., Brehme, C.S., Hines, J.E., Nichols, J.D. \& Fisher, R.N. (2012). Joint estimation of habitat dynamics and species interactions: disturbance reduces co-occurrence of non-native predators with an endangered toad. Journal of Animal Ecology, 81, 1288-1297.

Mordecai, R.S., Mattsson, B.J., Tzilkowski, C.J. \& Cooper, R.J. (2011). Addressing challenges when studying mobile or episodic species: hierarchical Bayes estimation of occupancy and use. Journal of Applied Ecology, 48, 56-66.

Nichols, J.D., Bailey, L.L., O’Connell Jr., A.F., Talancy, N.W., Campbell Grant, E.H., Gilbert, A.T., Annand, E.M., Husband, T.P. \& Hines, J.E. (2008). Multi-scale occupancy estimation and modelling using multiple detection methods. Journal of Applied Ecology, 45, 1321-1329.

Norrdahl, K. \& Korpimäki, E. (2000). The impact of predation risk from small mustelids on prey populations. Mammal Review, 30, 147-156.

Plummer, M. (2003). Jags: A program for analysis of bayesian graphical models using gibbs sampling. In: Proceedings of the 3rd international workshop on distributed statistical computing. Vienna, Austria., vol. 124, pp. 1-10.

R Core Team (2020). R: A Language and Environment for Statistical Computing. R Foundation for Statistical Computing, Vienna, Austria.

Richmond, O.M.W., Hines, J.E. \& Beissinger, S.R. (2010). Two-species occupancy models: a new parameterization applied to co-occurrence of secretive rails. Ecological Applications, 20, 2036-2046. 
Rota, C.T., Ferreira, M.A.R., Kays, R.W., Forrester, T.D., Kalies, E.L., McShea, W.J., Parsons, A.W. \& Millspaugh, J.J. (2016a). A multispecies occupancy model for two or more interacting species. Methods in Ecology and Evolution, 7, 1164-1173.

Rota, C.T., Wikle, C.K., Kays, R.W., Forrester, T.D., McShea, W.J., Parsons, A.W. \& Millspaugh, J.J. (2016b). A two-species occupancy model accommodating simultaneous spatial and interspecific dependence. Ecology, 97, 48-53.

Smith, M.M. \& Goldberg, C.S. (2020). Occupancy in dynamic systems: accounting for multiple scales and false positives using environmental dna to inform monitoring. Ecography, 43, 376386.

Soininen, E.M., Jensvoll, I., Killengreen, S.T. \& Ims, R.A. (2015). Under the snow: a new camera trap opens the white box of subnivean ecology. Remote Sensing in Ecology and Conservation, $1,29-38$.

Sutherland, C.S., Elston, D.A. \& Lambin, X. (2014). A demographic, spatially explicit patch occupancy model of metapopulation dynamics and persistence. Ecology, 95, 3149-3160.

Tabak, M.A., Norouzzadeh, M.S., Wolfson, D.W., Sweeney, S.J., Vercauteren, K.C., Snow, N.P., Halseth, J.M., Di Salvo, P.A., Lewis, J.S., White, M.D., Teton, B., Beasley, J.C., Schlichting, P.E., Boughton, R.K., Wight, B., Newkirk, E.S., Ivan, J.S., Odell, E.A., Brook, R.K., Lukacs, P.M., Moeller, A.K., Mandeville, E.G., Clune, J. \& Miller, R.S. (2019). Machine learning to classify animal species in camera trap images: Applications in ecology. Methods in Ecology and Evolution, 10, 585-590.

Tingley, M.W., Stillman, A.N., Wilkerson, R.L., Howell, C.A., Sawyer, S.C. \& Siegel, R.B. (2018). Cross-scale occupancy dynamics of a postfire specialist in response to variation across a fire regime. Journal of Animal Ecology, 87, 1484-1496. 
Waddle, J.H., Dorazio, R.M., Walls, S.C., Rice, K.G., Beauchamp, J., Schuman, M.J. \& Mazzotti, F.J. (2010). A new parameterization for estimating co-occurrence of interacting species. Ecological Applications, 20, 1467-1475.

Yackulic, C.B., Reid, J., Nichols, J.D., Hines, J.E., Davis, R. \& Forsman, E. (2014). The roles of competition and habitat in the dynamics of populations and species distributions. Ecology, 95, $265-279$. 